\title{
The first Sino-German symposium on cancer research, Shanghai May 2007
}

\author{
Yajun Guo • Manfred Dietel
}

Received: 27 July 2007 / Accepted: 27 July 2007 / Published online: 20 October 2007

(C) Springer-Verlag 2007

Keywords China - Germany · Sino-Germany Science Center

\section{Introduction}

This meeting report gives a short overview on the contributions of 17 leading scientists from China and Germany. The symposium was supported by the SinoGermany Science Center Bejing, the Deutsche Forschungsgemeinschaft and the National Science Foundation, China. It intended to foster communication and collaboration of researchers from both countries.

The "Migrating Cancer Stem Cell"-Concept was discussed by Thomas Brabletz (Dept. of Surgery Univ. of Freiburg). Most colorectal adenocarcinomas (CRC) have mutations in the APC gene leading to overexpression of the Wnt-pathway effector beta-catenin. By acting as a transcriptional activator, nuclear beta-catenin is decisively involved in two fundamental processes in embryonic development: epithelial to mesenchymal transition (EMT) and generation of stem cells (e.g., in intestine development).

\section{Y. Guo}

International Joint Cancer Institute, SMMU,

Shanghai, China

M. Dietel

Institute of Pathology,

Charité,

Berlin, Germany

\section{Dietel $(\square)$}

Department of Pathology, Humboldt University,

Campus Charité, Schumannstr. 20/21,

10117 Berlin, Germany

e-mail: manfred.dietel@charite.de
We have previously shown that nuclear beta-catenin accumulates in tumor cells at the invasive front undergoing an EMT. The amount of these cells strongly correlates with clinical outcome and metastasis formation. In contrast, tumor cells in central tumor areas are differentiated and often show membranous, E-cadherin-bound beta-catenin. Strikingly, also, metastases show again a differentiated phenotype lacking nuclear beta-catenin, indicating a regulatory role of the tumor environment for malignant progression.

Based on the developmental functions of beta-catenin and our data, we propose that both primary tumors and metastases are derived from the pool of EMT-associated "migrating cancer stem cells" at the tumor host interface, which are defined by strong nuclear beta-catenin accumulation and expression of its target genes. This gives these cells a feature, which drives malignant tumor progression including metastasis: The unusual combination of abilities allowing simultaneously to migrate and behave as a cancer stem cell.

Molecular Targets in Cancer Therapy-New Possibilities and Old Limitations were reported by Reinhard Buettner (Inst. of Pathology, University of Bonn). Increasing knowledge on molecular pathogenesis of malignant tumors and activated signaling pathways has led to the concept of targeted therapies. Small molecularly designed molecules target specifically oncoproteins, and thereby modulate growth, apoptosis, or invasion of cancer cells. Currently, most experiences exit with membrane-coupled growth factor receptors and their intracellularly coupled signaling kinases.

In cancer cells, these tyrosine kinase-triggered signaling pathways are frequently altered and causally involved in malignant transformation. Indeed, some of the tyrosine 
kinase-coupled receptors or their respective growth factors were originally cloned as retroviral oncogenes. Therefore, these critical oncogenic effector pathways represent attractive and often highly selective therapeutic targets. However, as experience with patients under long-term therapies with selective tyrosine kinase inhibitors accumulates, it becomes also evident that tumors develop escape strategies leading to resistance to therapy either via specific mutations or switches in intracellular signaling pathways.

We propose to discuss (1) current knowledge of the most frequently employed molecular targets and the strategies to design and develop specific inhibitors, (2) report first experiences with molecular diagnostics of resistance, and to (3) describe putative options for combinational therapies and second- or even third-line molecular therapies to address tumor-escape strategies in response to treatment. (4) Finally, we would also like to discuss implications on the health care system and reflect ethical considerations resulting from a science-based molecular medicine.

Reinhard Dengler (Dept. of Neurology Medical School) gave an overview on Modern Approaches in Functional Neurodiagnosis. A major goal of neurosurgery is to reduce the intervention-associated brain damage and functional deficits to a minimum. This holds especially true if brain tumors are localized in or close to important functional regions such as the eloquential or sensorimotor areas. Structural images may show the extension of the tumor and may allow for differentiation between tumor mass and brain edema, but do not provide precise information on the relation between the tumor and functional areas which may be entirely or partially involved or rather displaced by the space occupying lesion or may be even fully untouched.

In such situations, the neurosurgeon wishes a functionally oriented presurgical diagnosis to make the best strategy for the operation and to avoid as much unnecessary brain damage as possible. Modern functional imaging in conjunction with specific structural imaging is very valuable in solving this task. In addition to classical cranial computer tomography and magnetic resonance imaging (MRI) and its variations including Gadolinium enhancement, functional MRI, and PET studies using tracers such as F-18-Deoxyglucose or C-11-Methonin (or F-18-Tyrosin) can be used successfully. Matching of the images generated by these techniques provides information on tumor extension, metabolically active tumor portions vs inactive or necrotic portions, and anatomical relationship to functional areas. The power of these techniques can be further increased by modern neuronavigation which uses the computerized imaging data. This helps the surgeon to make optimal intraoperative decisions on how much tissue can or must be removed and to minimize postoperative functional deficits.

Manfred Dietel (Inst. of Pathology, Charitè, Berlin) discussed Molecular Pathology and Targeted Therapy. To read a patients' tissue as "deeply" as possible and to get information on morphological, genetic, proteomic, and epigenetic grounds will be the upcoming task of surgical pathologists to provide the clinicians with information relevant for individualized medicine. These data will provide the basis of clinical drug selection as well as development of new drugs.

Molecular diagnostic Following the WHO, malignant tumors are grouped on the basis of histogenesis, histological type, and differentiation. However, tumors of the same group may be heterogeneous. Although histopathology is only partly able to predict clinical behavior, it still plays the dominant role in selecting patients' management. To improve this situation, molecular approach will become part of the diagnostic process, hopefully adding that information which conventional morphology is not able to provide.

Predictive pathology Prediction of the clinical behavior of an individual tumor is a pushing challenge for pathology. Clinicians strongly demand for a reliable prediction regarding prognosis, metastatic potential, and/or response to therapy. Applying DNA arrays, several approaches have been performed successfully to improve predictive accuracy.

Development of new drugs In recent years, the efforts devoted to unraveling potential new therapeutic targets have lead to the development of new drugs. For example, Herceptin and Gleevec are the first designer drugs which are used with considerable success in the clinic. The basis of these developments was provided by tissue-based investigations performed by experimental pathologists.

Bernd Groner (Georg Speyer Haus, Inst. for Biomedical Research, Frankfurt) presented data on "Novel Approaches to Targeted Tumor Therapy". The inappropriate assembly of modular components plays a role in oncogenic transformation and other diseases and can be exploited for therapeutic purposes. These target structures, however, are conventionally considered as "not druggable". As proteinprotein interactions are mediated by surface structures of folded proteins, peptides embedded in the context of a scaffold protein can serve as competitive inhibitors of protein recognition modules. Such peptide aptamers can target non-canonical drug binding sites and disrupt intracellular protein functions. Applications of this principle are dependent upon the binding specificities and affinities of the peptide aptamers to their target structures, recombinant expression, and purification of the peptide aptamer-scaffold protein and delivery of the purified protein into cells. We improved upon the scaffold structure and utilized the 
human thioredoxin molecule. Removal of five internal cysteines allows the purification of a monomeric protein with high yield. Provision of this molecule with a protein transduction domain, consisting of nine arginines, results in a fusion protein, which is taken up efficiently by cultured cells, inhibits intracellular signaling functions and associated cellular phenotypes. A peptide aptamer which recognizes the intracellular part of the ErbB2 receptor was introduced into breast cancer cells. They reduced the extent of AKT kinase induction upon heregulin treatment of the cells and enhanced resistance towards chemotherapeutic agents in ErbB2 overexpressing cancer cells. A peptide aptamer directed against the dimerization domain of the transcription factor Stat3 prevented Stat3-mediated transactivation and induced growth inhibition and the induction of apoptosis.

Christian Hagemeier (Charité-Berlin, Children's Hospital, Lab for Molecular Biology, Berlin) spoke about Molecular Approaches to Understand and Tackle Acute Lymphoblastic Leukemias in Children. The probability of long-term survival after relapse from acute lymphoblastic leukemia (ALL) is predicted from well-established prognostic, mainly clinical, factors. However, the underlying biological determinants of these prognostic factors are poorly understood. In collaboration with the department of Pediatric Oncology that houses the BFM ALL-REZ study group, we have defined molecular prognostic markers for risk stratification, identified gene expression programs that characterize ALL pathogenesis in patients with poor outcome, and searched for genes that modulate therapeutic approaches. We found that early onset relapse of ALL, which is typically associated with a particularly low eventfree survival rate, is distinguished from late disease recurrence by an increased proliferative capacity of leukemic blasts and a distinct expression of genes with functions in cell cycle control, particularly during the process of mitosis. In addition, by employing a genetic shRNA screen, we have identified sets of genes that modulate therapeutic approaches in cell culture-based models. The implications will be discussed.

Per Sonne Holm (Inst. of Experimental Oncology \& Research, Technical University Munich) reported on YB-1 as a Target for the Development Oncolytic Adenovirus. It has been shown that YB-1 regulates the expression of a number of genes linked to cell proliferation and growth. In addition, YB-1 is a multifunctional protein involved in regulating translation, mRNA splicing, and DNA repair, indicating that YB-1 is involved in controlling cell survival. In recent years, several laboratories have demonstrated that YB-1 in response to genotoxic stress like chemotherapy translocates from the cytoplasm to the nucleus. Besides transcriptional activation of the human MDRI and MRPI genes, nuclear localization of the transcription factor YB-1 is associated with base excision and mismatch repair pathways through binding to several DNA repair proteins. YB-1 is found amongst others highly overexpressed in many malignancies, and current results suggest that YB-1 is important in conferring, e.g., drug resistance in many types of tumor cells. Oncolytic adenoviruses have attracted considerable attention for their use as an innovative class of promising cancer therapeutics. We have developed several oncolytic adenoviral vectors which replicate in the presence of YB-1 in tumor cells. The application of YB-1dependent oncolytic vectors produces a strong antitumor effect in several carcinoma cell lines, and application in vivo in subcutaneous established tumors led to strong tumor growth inhibition, which could further be enhanced by radiation and chemotherapy. In conclusion, YB-1dependent adenoviruses are highly effective against different malignancies and deserve further clinical testing.

Reinhold Schäfer (Inst. of Pathology Charité Berlin) explained Oncogenic Ras Signaling: Effector Pathways, Targets, Regulation and Interference. Hundreds of transcriptional targets are up-regulated or down-regulated on transition from the normal to the Ras-transformed state. A minority of targets is equally deregulated in different cell types, indicating that there exist a limited number of common Ras pathway targets. The set of up-regulated genes comprises factors involved in growth control, invasion, and metastasis, while many down-regulated genes encode proteins with anti-proliferative, anti-invasive, and antiangiogenic properties. The involvement of most of the deregulated genes in triggering or executing various aspects of malignant transformation is unknown. Moreover, the global mechanisms controlling their expression are not yet understood. To unravel the regulatory mechanisms perturbing the transcriptional networks downstream of the Ras signaling cascade and to find out which of the targets are primarily involved in tumorigenesis, we are employing several experimental strategies: We have narrowed down the number of critical targets by correlating the effects of blocking individual effector pathways, using pharmacological inhibitors or RNA interference, with the transcriptional patterns and specific phenotypes. We have selected a few targets exhibiting significant differential expression in Rastransformed vs normal cells for RNA interference experiments. To study the role of DNA methylation in target gene suppression, we subjected Ras-transformed cells to treatment with demethylating agents and determined the fraction of responding targets. As an example, we have shown that clusterin gene suppression is controlled by promoter methylation and by the MAPK-pathway, suggesting a dynamic relationship between Ras signaling and DNA methylation.

Unraveling the relationships between effector pathways and transcriptional networks will provide important clues for understanding the system biology of cancer, help to 
identify novel biomarkers, and eventually, more specific nodes for therapeutic intervention.

Xuetao Cao (Inst. of Immunology \& National Key Laboratory of Medical Immunology, SMMU, Shanghai) gave a report on Nuclear Entrapment of MEK1/2 by an Oncogenic Ras-Related GTP-Binding Protein Promotes Tumor Progression. Tumorigenesis is widely recognized as an intricate multistep process that involves malfunction in protooncogenes, tumor suppressor genes, and other key cellular genes implicated in cell proliferation, differentiation, survival, and genome integrity. Malfunction of protooncogenes and signaling components has been reported. Among them, the Ras/Raf/MEK [mitogen-activated protein kinase (MAPK)/ERK kinase]/ERK (extracellular signal-regulated kinase) signaling components have been identified to play important roles in tumorigenesis. MEK1/2 are cytoplasmic proteins containing nuclear export signal (NES) and play crucial roles in determining cell growth and differentiation through activation of extracellular signal-regulated kinase (ERK1/2).

RJLs are a family of Ras-related GTP-binding proteins that are characterized by the N-terminal GTP-binding domain (small GTPase domain) and the C-terminal $\mathrm{J}$ domain with unknown functions. The full-length human RJL (hRJL) cDNA was identified by us via large-scale sequencing of a human peripheral blood monocyte-derived dendritic cell cDNA library. Here, we report that hRJL, a nucleus-localized representative of RJL family, can mediate the accumulation of active MEK1/2 in the nucleus by masking the NES of MEK1/2, which in turn results in constitutive activation of ERK $1 / 2$ and malignant transformation. We show that hRJL is overexpressed in most of the tumor cells and tumor tissues and is oncogenic both in vitro and in vivo. Silencing of hRJL expression or disruption of hRJL-MEK1/2 interaction results in regression of tumor growth and metastasis. Our findings suggest that hRJLmediated nuclear entrapment of MEK $1 / 2$ plays important roles in promotion of autonomous tumor progression, and hRJL is a novel oncogenic therapeutic target.

Guo-Qiang CHEN (Dept. of Pathophysiology, Shanghai Jiao Tong University School of Medicine) reported on Hypoxia and Leukemic Cell Differentiation, Evidence From In Vitro and In Vivo. He stated that cobalt chloride and desferrioxamine-mimicked hypoxia and moderate hypoxia can trigger acute myeloid leukemia (AML) cells to undergo differentiation, and intermittent hypoxia significantly prolongs the survival of the transplanted leukemic mice through inducing growth arrest and differentiation with inhibition of leukemic blast infiltration. Based on these in vitro and in vivo observations, we explore the role of hypoxia-inducible factor 1(HIF-1), a protein in leukemic cell differentiation. Our results show that the conditional HIF-1a induction triggers granulocytic differentiation of myeloid leukemic U937T transformants in which HIF-1a is tightly induced by tetracycline withdrawal. The suppression of HIF-1a expression, but not HIF-1b (an essential partner for transcription activity of HIF-1), by specific short hairpin RNAs (shRNAs) effectively inhibits hypoxia-induced differentiation of U937 cells. Further, the inhibition of hematopoietic differentiation-critical CCAAT/enhancer binding protein-alpha $(\mathrm{C} / \mathrm{EBPa})$ significantly eliminates HIF-1a-mediated myeloid leukemic cell differentiation. Furthermore, HIF-1a is also shown to interact physically with and to increase the transcriptional activity of $\mathrm{C} / \mathrm{EBPa}$ and AML1, two critical factors for granulocytic differentiation. Collectively, this work provides several lines of direct evidence for the role of HIF-1a protein through its nontranscriptional activity in myeloid cell differentiation in which $\mathrm{C} / \mathrm{EBPa}$ elicits a role as an effector downstream to HIF-1a. These discoveries would shed new insights for understanding mechanisms underlying leukemogenesis and designing the new therapeutic strategy for differentiation induction of AML.

Jing Cheng (Medical Systems Biology Research Center, Tsinghua University School of Medicine Haidian District, Beijing) explained Diagnostic Biochips: From Research to Consumer Market. Biochip-based platforms for research have developed rapidly in the past decade, which includes chips that can generate detailed biological information at four different levels - at the gene, protein, cell, and tissue levels. For example, at the gene level, we have SNP and mutation analysis microarrays, comparative genome hybridization microarrays, methylation detection microarray, and mRNA and miRNA expression profiling microarrays; at the protein level, we have established activated transcription factor profiling chips as well as ChIP-GLAS-based promoter chips; at the cellular level, we have chips for single cell ion flux monitoring and multichannel electrophysiology measurement of cell networks; and finally, for pathological examination, we can provide numerous different tissue microarrays, including normal and diseased tissues.

In recent years, demand for general consumption of these new classes of biochips has been keenly anticipated in consumer areas such as disease diagnostics, biosafety testing, etc. Furthermore, in this presentation, different types of consumer biochip products will be introduced, including DNA microarrays for the detection of the presence of SARS Corona virus RNA, for detection of Gram-positive bacteria and the associated drug resistance genes, for detection of mycobacterium and the drug resistance genes for $\mathrm{TB}$, for detection of food-borne pathogen, and protein microarrays for the diagnosis of autoimmune diseases, and for the detection of veterinary drug residues such as antibiotics. This broad range of applications for biochips foreshadows future development of a wider range of sensitive testing products. 
Jian-ren Gu (State Key Lab. of Oncogenes and Related Genes, Shanghai Cancer Institute, Jiao Tong University) discussed Conceptual Concerns on Cancer Biology. The major problems in cancer treatment are:

1. Cancer is not a cluster of cancer cells, but a wellorganized tissue.

2. Cancer cells are heterogeneous. Among cancer cell population, the cancer stem cells may be crucial targets for treatment.

3. Dissemination may be an early event in most types of cancer, also a different event from metastasis. The mechanism to evoke the growth of disseminated cancer cells into a metastatic lesion is crucial in development of new therapeutic methods to the control of cancer.

4. Cancer could be a systems disease with local manifestation of abnormal cell growth developed on the basis of deregulation of host-cell homeostasis, including neural, humoral, and immune mechanism.

These conceptual considerations may provide some clues to explore the mechanism of carcinogenesis and cancer progression as well as the design of new therapeutic strategy against cancer.

Yajun Guo (International Joint Cancer Institute, SMMU, Shanghai, yjguo@smmu.edu.cn) explained the topic of "Novel Vaccine Development and Cancer Immunotherapy". We developed several novel bispecific antibodies, which consist of two normal binding sites for targeting antigens and a functioning portion of Flt3L. The constructed bispecific antibodies (SM5.1-Flt3L, Anti-her2-Flt3L, and anti-CD20-Flt3L) efficiently target hepatoma, breast, and lymphoma cancer cells both in vitro and in vivo, leading to accumulation of DCs, NK cells, and lymphocytes in local tumor tissues. Administration of these bispecific monoclonal antibodies can protect bone marrow injury caused by chemotherapeutic drugs and stimulates proliferation and maturation of lymphocytes, APCs, and NKs. Systemic administration of these bispecific antibodies $(100 \mu \mathrm{g} /$ each injection $\times 3$ ) significantly inhibited tumor growth and cured established tumors. These bispecific therapeutic proteins are effective in elicitation of long-lasting antitumor immunity. The tumor-specific immunity can be adoptively transferred into naïve animals successfully by transfusion of $\mathrm{CD}^{+} \mathrm{CD}^{+} \mathrm{T}$ cells from the treated mice. The results suggest that fusion of Flt3L with therapeutic antibodies can create novel immunotherapeutic proteins, which are more effective in induction of antitumor immunity, and may provide a novel strategy for treatment of cancers.

Yongfeng Shang (Peking University) talked about Gene Regulation by Nuclear Receptors. Steroid receptor coactivators (SRCs) exert profound effects on animal development and physiology. These coactivators are nuclear proteins and transcription co-regulators that function to facilitate the transcription initiation mediated by nuclear receptors as well as by other well-known transcription factors. However, how these co-regulators are functionally regulated is poorly understood. During genome-wide screening for SRC-interacting proteins, we identified a novel ankyrin-repeat-containing protein, SRC-interacting protein (SIP), which interacts with SRC coactivators in the cytoplasm. We demonstrated that extracellular stimuli, such as the addition of estrogen, induced phosphorylation of SIP in its PEST (proline, glutamate, serine, and threonine-rich) domain by casein kinase II. The phosphorylation of SIP resulted in dissociation of SRC proteins from SIP in the cytoplasm and led to subsequent nuclear translocation of SRC proteins and gene coactivation. Both gain-of-function and loss-of-function experiments indicate that SIP functions to sequester SRC coactivators in the cytoplasm and buffer the availability of these coactivators, thus, providing a mechanism for the regulation of the transcription regulators.

Hongyang Wang (Inst. International Cooperation Laboratory on Signal Transduction Eastern Hepatobiliary Surgery Institute/Hospital, Shanghai SMMU) Negative Regulation of Hepatocellular Carcinoma Cell Growth by Signal Regulatory Protein. $1 \mathrm{SIRP} \alpha 1$ is a member of the signal regulatory protein (SIRP) family that undergoes tyrosine phosphorylation and binds SHP-2 tyrosine phosphatase in response to various mitogens. The expression levels of $\operatorname{SIRP} \alpha 1$ were decreased in human hepatocellular carcinoma (HCC) tissues, as compared with the matched normal tissues. Exogenous expression of wild-type $\operatorname{SIRP} \alpha 1$, but not of a mutant $\operatorname{SIRP} \alpha 1$ lacking the tyrosine phosphorylation sites, in SIRP $\alpha 1$ negative Huh7 human HCC cells resulted in suppression of tumor cell growth both in vitro and in vivo. Treatment of Huh7 transfectants with EGF or HGF induced tyrosine phosphorylation of SIRP $\alpha 1$ and its association with SHP-2, which were accompanied by reduced ERK1 activation. Expression of $\mathrm{SIRP} \alpha 1$ significantly suppressed activation of NF- $\mathrm{KB}$ and also sensitized Huh7 cells to TNF $\alpha$ or cisplatin-induced cell death. In addition, SIRP $\alpha 1$-transfected Huh7 cells dis played reduced cell migration and cell spreading in a fashion that was dependent on SIRP $\alpha 1 /$ SHP- 2 complex formation. In conclusion, these results suggest a negative regulatory effect of SIRP $\alpha 1$ on hepatocarcinogenesis through, at least in part, inhibition of ERK and NF-KB pathway. The heightened sensitivity of cells restoring SIRP $\alpha 1$ function could be exploited in the development of therapeutic regimens which may potentiate the antineoplastic effect of conventional cytokines or chemotherapeutic agents.

Qimin Zhan (Cancer Institute Chinese Academy of Medical Sciences) explained the role of Bacp, a BRCA1Regulated Centrosomal Protein, in the Control of Genomic 
Stability. The essence of successful mitosis in mammalian cells is the generation of two genetically identical daughter cells. The successful mitosis requires the assembly of a strictly bipolar mitotic apparatus that will ensure that chromosomes equally distribute to the daughter cells. This process is controlled by the centrosomes that are required for spindle formation and function. Centrosome stability is required for the successful mitosis in mammalian cells. Amplification of centrosome leads to chromosomal missegregation and generation of aneuploidy, which are closely associated with cell transformation and tumorigenesis. The machinery that controls centrosome stability involves multiple important cellular proteins, including p53, BRCA1, Gadd45, p21, and Cdk2/cyclin E. The precise coordination among those regulators maintains centrosome duplication and stability. However, there are currently limited insights into mechanism(s) for this critical biological event.

Breast cancer susceptibility gene BRCA1 is implicated in the control of mitotic machinery, although there is little insight into underlying mechanism(s). Here, we show that BRCA1 physically interacts and co-localizes with Bacp (BRCA1 associated centrosomal protein). Interestingly, Bacp centrosomal localization likely depends on normal cellular BRCA1 function, as cells containing BRCA1 mutations or silenced for endogenous BRCA1 reveal disrupted Bacp co-localization to centrosomes. Suppression of endogenous Bacp results in aberrant spindle formation, failure of chromosomal segregation and cytokinesis, and aneuploidy. Bacp is overexpressed in human breast and lung carcinomas, and its deregulation is in part associated with $B A C P$ amplification. Bacp exhibits strong oncogenic property and induces NIH3T3 fibroblast transformation. Importantly, Bacp transgenic mice mimic the phenotypes of disrupted BRCA1, including centrosome amplification and spontaneous tumorigenesis. Thus, Bacp may cooperatively act together with BRCA1 in mitotic machinery, and abnormalities of Bacp lead to genomic instability and tumorigenesis.

Jingde Zhu (Cancer Epigenetics and Gene Therapy Program, Shanghai Cancer Institute) Cancer Epigenetics (-omics) from DNA Methylation Perspectives in Liver Cancer as a Paradigm Cancer is a complex disease of broad genetic and epigenetic defects. The epigenetic circuit dictates the inheritable transcription profile and therefore the phenotype, better understanding of which would revolutionize the current anti-cancer practice. Epigenetic makeup comprises DNA methylation, histone modification, microRNAs, and chromatin remodeling, which act together to shape the chromatin structure of genome to meet the functional requirements. DNA methylation, an enzymatic process to add a methyl group at the fifth carbon of cytosine within the palindromic dinucleotide $5^{\prime}$-CpG-3' sequence is the best-studied pigenetic mechanism. Cancerassociated aberration of DNA methylation goes two opposite directions: global hypomethylation and local hypermethylation, that, in turn, dictate the transcription profile of both repetitive sequences and individual genes, and therefore, the phenotypic profiles unique to cancer states HCC present a major health threat to the people in China, owing to their high incidence and aggressive clinical nature. We are currently carrying out comprehensively profiling the disease-associated $\mathrm{CpG}$ methylation pattern at the multiple gene level (1) and at the global level (2) to gain the new mechanistic insights, establish a DNA methylationbased staging and classification system and find effective biomarkers for HCC. To demonstrate the great potential of DNA methylation in cancer detection, I will present the latest results in DNA methylation profiling in urine sediments for sensitive/specific detection of bladder cancer. 